\title{
Picture the difference: electrophysiological investigations of picture processing in the two cerebral hemispheres
}

\author{
Kara D. Federmeier ${ }^{\mathrm{a}, *}$, Marta Kutas ${ }^{\mathrm{a}, \mathrm{b}}$ \\ ${ }^{a}$ Department of Cognitive Science, University of California, San Diego, 9500 Gilman Dr., La Jolla, CA 92093-0515, USA \\ ${ }^{\mathrm{b}}$ Department of Neurosciences, University of California, San Diego, 9500 Gilman Dr., La Jolla, CA 92093-0515, USA
}

Received 14 July 2000; received in revised form 2 August 2001; accepted 4 September 2001

\begin{abstract}
The nature of semantic memory and the role of the two cerebral hemispheres in meaning processing were examined using event-related brain potentials (ERPs) elicited by pictures in sentences. Participants read sentence pairs ending with the lateralized presentation of three target types: (1) expected pictures, (2) unexpected pictures from the expected semantic category, and (3) unexpected pictures from an unexpected category. ERPs to contextually unexpected pictures were more negative 350-500 ms (larger N400s) than those to expected pictures in both visual fields. However, while N400s to the two types of unexpected items did not differ with left visual field presentations, they were smaller to the unexpected items from the expected category with right visual field presentations. This pattern, previously observed to words [Brain Language 62 (1998) 149], suggests general differences in how the two hemispheres use context on-line. Other aspects of the N400 response-and effects on earlier ERP components-reveal differences between pictures and words, suggesting that semantic memory access is not modality-independent. The P2 component varied with ending type for right but not left visual field presentations, suggesting that the left hemisphere may use contextual information to prepare for the visual analysis of upcoming stimuli. Furthermore, there was clear evidence for an earlier negativity ("N300"), which varied with ending type but, unlike the N400, was unaffected by visual field of presentation. Overall, the results support our hypothesis that the left hemisphere actively uses top-down information to preactivate perceptual and semantic features of upcoming stimuli, while the right hemisphere adopts a "wait and see" integrative approach. (C) 2002 Elsevier Science Ltd. All rights reserved.
\end{abstract}

Keywords: Hemispheric differences; Semantic memory; Sentence processing; N400; N300

\section{Introduction}

Sensory information from the ear, eye, and body surface is divided such that part of the signal from each modality impinges, at least initially, preferentially on one of the two cerebral hemispheres. Lateralized differences in, for example, neuronal number and connectivity (e.g. $[25,26,61])$ as well as in neurotransmitter distribution and sensitivity to hormones (e.g. $[42,65])$ then cause these sensory inputs to be analyzed somewhat differently in the two hemispheres. Given that the two hemispheres seem to perceive the world somewhat differently, a question of long-standing interest is whether there are also hemispheric-specific records of information in long-term memory and, if so, how the content and organization of the semantic knowledge base in each hemisphere might differ. The nature of long-term semantic memory in the two hemispheres, in turn, has important im-

\footnotetext{
* Corresponding author. Tel.: +1-858-34-2440; fax: +1-858-534-1128. E-mail address: kfederme@cogsci.ucsd.edu (K.D. Federmeier).
}

plications for how each might process language and, indeed, make sense of the world more generally.

Studies using visual half-field techniques have in fact uncovered a number of differences in how the hemispheres respond to individual words and use verbal context information in real time. For example, while word-pair priming based on close semantic relationships (e.g. DOG-CAT) is equivalent in the two hemispheres, priming of more distantly-related items (e.g. DOG-GOAT) seems to be restricted to the right hemisphere $[8,10,12]$. This has led to the hypothesis that meaning activation in the left hemisphere is biased toward close lexical-semantic relationships while meaning activation in the right hemisphere is broader in scope. Others have suggested that this difference in scope arises because the right hemisphere activates semantic information more slowly than the left and maintains activation over longer time periods [5,6,34]. Results from studies using phrases or sentences instead of word pairs have led to the additional suggestion that the left hemisphere makes much greater use of message-level context information. For example, right hemisphere processing has been reported to be less sensitive 
than left hemisphere processing to semantic and syntactic congruity (e.g. [19], but see also [11]) and to sentential constraint [20].

To test some of these hypotheses, we used electrophysiological methods to examine hemispheric differences in semantic memory organization and in the use of sentence context information on-line [21]. In addition to providing a link to the neurobiology of behavior, electrophysiological methods have millisecond-level temporal resolution and allow inferences about both qualitative and quantitative processing differences. Use of electrophysiological methods also obviates the need to employ tasks requiring a speeded response, most of which are performed with differential efficiency by the two hemispheres. In our study, participants read for comprehension sentence pairs (e.g. "They wanted to make the hotel look more like a tropical resort. So along the driveway they planted rows of ...") completed by one of three types of target words, presented to the left and right visual-half-fields: (1) expected exemplars, the best completion for a particular sentence pair (e.g. "palms"), (2) within category violations, unexpected completions derived from the same semantic category as the expected ending (e.g. "pines"), and (3) between category violations, unexpected completions from a different semantic category (e.g. "tulips"). We measured the amplitude of the N400, a negative-going potential peaking around $400 \mathrm{~ms}$ that has been linked to semantic processing (for review, see [37]), in response to the three types of target words as a function of visual field of presentation.

Right hemisphere initiated responses to these target words showed a sensitivity only to sentence context information; expected exemplars generated less N400 activity than unexpected completions of either type (which did not differ). In contrast, left hemisphere initiated responses reflected the influence of both context and the context-independent semantic similarity of the items; while expected exemplars generated less N400 activity than unexpected items overall, within category violations, which share semantic features in common with the expected item, generated smaller N400s than between category violations. These results are taken to suggest differences in how the two hemispheres use context on-line. While the right hemisphere seems to integrate new information directly with context information held in working memory, the left hemisphere seems to use context to preactivate the features of upcoming items and to compare new information with that "prediction", rather than with context information directly.

Both behavioral and electrophysiological studies of word processing have thus suggested that the hemispheres differ in their use of semantic information on-line. A few studies have also examined hemispheric differences in the semantic processing of pictures. Studies of picture processing in the two hemispheres provide an interesting counterpart to studies of word processing for a number of reasons. First, the use of pictures circumvents the concerns raised by left hemisphere dominance for word recognition $[47,57]$ and for language processing more generally. Levine and Banich [41] showed, for example, that while the typical left hemisphere advantage is observed for word reading, no lateralized differences are found for the naming of pictures of the same objects (see also Experiment 1 of Biederman and Cooper [4]). Similarly, no hemispheric differences were observed in a picture-name priming study [16]. Studies with commissurotomized individuals have indicated that both isolated hemispheres can match pictures on the basis of class inclusion and part-whole relationships [72] as well as on the basis of even more abstract conceptualizations (e.g. a gun and a black eye as symbols of violence) [17]. The hemispheres thus seem to be more equally matched in their ability to process pictorial stimuli, making it correspondingly easier to address hypotheses relating to semantic activation and integration when pictures, rather than words, are used.

While both hemispheres seem to be able to derive semantic information from pictures, it is also clear that each processes visual stimuli differently in a number of ways. Differences in reaction times and/or error rates as a function of visual field have been observed for stimuli varying in features such as retinal eccentricity (angle away from the visual midline), size, luminance, contrast, exposure duration, and spatial frequency (reviewed in [14,15]). In general, left hemisphere performance seems to be preferentially impaired relative to right hemisphere performance (which may even be improved) by increasing retinal eccentricity and size and by decreasing luminance, contrast, exposure duration, and spatial frequency (at least for non-verbal stimuli, see [13]). Moreover, the hemispheres also seem to differ in the extent to which they process visuo-spatial relations in categorical (e.g. above/below) or metric (distance) terms (e.g. $[1,29,36,40,60])$, with left hemisphere processing more categorical and right hemisphere processing more metric in nature. Finally, the hemispheres also seem to differ in the extent to which they direct attention to global (whole object) or local (object part/feature) aspects of visual stimuli (e.g. $[18,24,66])$; in general, processing in the left hemisphere seems more directed to local stimulus properties and processing in the right hemisphere to global stimulus properties. Such attentional differences may also underlie observed differences in the hemispheres' abilities to remember information conveyed in pictures: the right hemisphere's memory seems to be more sensitive to global scene organization and coherence, while the left hemisphere seems more proficient at remembering unorganized scenes or incongruent details in scenes [70,71]. What these perceptual and short-term memory differences imply for semantic processing in the two hemispheres remains an open question, providing yet another reason to study how the two hemispheres treat pictures at both perceptual and semantic levels.

Finally, it is interesting to compare hemispheric differences for picture processing with those for word processing because similarities and differences in word and picture processing have long been used more generally to assess models of semantic memory. Whereas "common semantic 
system" or "single code" models hypothesize that words and pictures converge upon a single, amodal semantic store (e.g. $[7,28,54,56,58,63])$, "multiple semantic system" or "dual code" models suggest instead that pictures and words are processed in distinct, specialized semantic systems (e.g. [51-53,62]). Consideration of hemispheric differences in word and/or picture processing-something most extant models do not deal with explicitly-opens a number of interesting questions. If there is a common, amodal semantic system, is it distributed across both hemispheres or are there two amodal stores? If semantic knowledge is compartmentalized, is it split by hemisphere as well as modality (Paivio [52], for example, has suggested that word-based semantics is located in the left hemisphere while picture-based semantics is located in the right hemisphere)? Is it possible for one hemisphere's semantic processing to be amodal while the other's processing is modality-specific? Clearly, a complete model of semantic memory will need to explicate how semantic knowledge is represented as a function of both modality and hemisphere.

It is thus plainly of interest to use both word and picture stimuli to examine semantic memory activation and organization in the two cerebral hemispheres; however, only a handful of such studies exist for picture stimuli. Three studies have examined semantic priming with picture-picture pairs. Using a naming task, Biederman and Cooper [4] found no hemispheric differences in the semantic priming of basic-level category exemplars (e.g. grand piano and upright piano). In contrast, Hines et al. [31] (also using a naming task) observed priming for categorically-related items only in the left hemisphere. The stimuli used by Hines et al. seemed to be less closely related than those used by Biederman and Cooper (though Hines et al. do not list all of their stimuli); assuming this is true, the different pattern of results could be taken as suggestive of broader meaning activation from pictures in the left than in the right hemisphere, opposite to the pattern observed for words. Zaidel [69], using a category membership decision, found that typical pairs (e.g. apple and banana) were processed more quickly than atypical pairs (e.g. watermelon and boysenberries) in the right hemisphere, while the left hemisphere's responses did not vary as a function of typicality. These results were taken to imply that, for picture stimuli, the right hemisphere maintains a more fine-grained category membership structure; again, this goes in the opposite direction from proposals that have been put forward to explain word priming results (e.g. [3]).

There is thus some indication that the nature of semantic processing of pictures in the two hemispheres may differ from the semantic processing of words (see also [9]). It is difficult, however, to draw clear conclusions from such a small number of studies, especially since none have compared word and picture processing using equivalent sets of stimuli and task conditions. We, therefore, decided to examine picture processing in the two hemispheres using the same task conditions, sentence contexts, and target concepts that we used previously to study word processing [21], here replacing target words with line drawings representing the same objects. Such a design allows more specific comparisons between word and picture processing in the hemispheres than have been possible to date. To our knowledge, this is the first study of any kind to examine lateralized differences for the processing of pictures in sentence contexts (a design pioneered by Potter et al. [55]) and the first to use event-related brain potentials (ERPs) to examine picture processing in a visual half-field design.

Previous ERP work has shown that, like the semantic analysis of words, the semantic analysis of pictures presented centrally is associated with changes in a negativity peaking $250-500 \mathrm{~ms}$ post-stimulus onset $[2,27,32,38,49]$. This "picture N400" has a similar time course as the N400 observed to words and shows a similar reduction in amplitude in response to pictures in congruent as opposed to incongruent contexts [27,49]. However, the distribution of the picture and word N400s are not identical; while the N400 to words is maximal over central-posterior sites, the $\mathrm{N} 400$ to pictures shows a more frontal distribution [27]. This may suggest that the neural generators responsible for this component in the two cases are not identical. Alternatively, McPherson and Holcomb [46] have argued that the observed distributional difference is due to the partial overlap, in the case of pictures, of the N400 by an earlier, frontally-distributed negativity ("N300"). The precise functional significance of this component remains to be elucidated; McPherson and Holcomb suggest that it is related to the processing of "object-specific information". They found that the N300 was less sensitive than the N400 to the degree of semantic overlap between two items but more sensitive than the N400 to object structure information (difference between pseudo-objects, which violate structural principles, and scrambled objects, which are non-sensical but possible) (see also [32,45]).

Recently we examined the processing of pictures in central vision, comparing the response to expected exemplars, within category violations, and between category violations (as described previously) in highly constraining and less constraining contexts [23]. At a general level, the response to pictures was functionally similar to that previously observed with the presentation of words in central vision [22]: expected exemplars elicited smaller N400 responses than violations of either type, but within category violations elicited smaller N400s than between category violations. Thus, there seems to be an overall similarity not only in how pictures and words are integrated into a sentence context but also in the organization of the semantic knowledge base tapped into by pictures and words. However, there are also some modality-based processing differences, as contextual strength differentially affected the semantic integration of words and pictures. While we observed two negative peaks in the $250-500 \mathrm{~ms}$ time window (N300 and N400), we did not find differences in the N300 and N400 responses to the experimental variables. Overall, the pattern of results suggests that while pictures and words may share 
a semantic system, access to this system is not completely modality-independent.

In this experiment, therefore, we examine the processing of expected pictures, unexpected pictures that share many semantic features in common with the expected picture (within category violations), and unexpected pictures that do not share many features in common (between category violations). We ask whether the pattern of hemispheric differences we observed for word processing in the same sentence contexts [21] also holds when the same target concepts are represented by line drawings, suggestive of a general (modality-independent) difference in how the two hemispheres use context information on-line. If this is the case, then we expect to find that the N400 response to pictures presented initially to the right visual field (left hemisphere) will vary with both congruency and category membership, while that to items presented initially to the left visual field (right hemisphere) will vary only with congruency. ${ }^{1}$ Alternatively, it may be that the modality differences we have observed between word and picture processing in central vision $[22,23]$ will interact with hemispheric differences, yielding a different pattern of effects in the N400 responses to pictures as a function of visual field of picture presentation than was previously observed for words. We also examine earlier ERP components (N1, P2, and N300) related, respectively, to attentional allocation, visual feature detection, and (possibly) object recognition in order to look for hemispheric differences in the perceptual analysis of pictures and to see how such differences, if observed, relate to later, semantic effects.

\section{Methods}

\subsection{Materials}

Stimuli were derived from those used in Federmeier and Kutas [21]. They consisted of 132 pairs of sentences, ending with three types of targets: (1) expected exemplars, items with the highest cloze probability in the sentence contexts, (2) within category violations, contextually unexpected items derived from the same taxonomic category as the expected exemplar, and (3) between category violations, contextually unexpected items derived from a different category than the expected exemplar. Table 1 gives examples. Sentence-final words in the Federmeier and Kutas studies were replaced in this experiment with black and white line drawings derived from the Snodgrass and Vanderwart set [64] and from a commercial clip art package; these were the same line drawings as were used in Federmeier and Kutas [23]. Each line drawing was normed to ensure naming agreement with the word that it would replace. Nineteen individ-

\footnotetext{
${ }^{1}$ Note that we examine laterality issues in this study by looking at differences in the pattern of response to experimental variables as a function of visual field of presentation. Our predictions and our subsequent interpretations are not based on scalp distribution effects.
}

Table 1

Example stimuli (expected exemplar/within category violation/between category violation)

The firefighters wanted to have a mascot to live with them at the firehouse Naturally, they decided it would have to be a dalmatian/poodle/zebra.

Muffie, old Mrs. Smith's pet, wears a bow on the puff of fur on its head I do not know how anyone could want to own a poodle/dalmatian/donkey.

"I am an animal like Eeyore!" the child exclaimed

His mother wondered why he was pretending to be

a donkey/zebra/dalmatian.

At the zoo, my sister asked if they painted the black and white stripes on the animal.

I explained to her that they were natural features of a zebra/donkey/poodle.

uals were asked to name each picture; pictures were used in the study only if the replaced word was the most commonly named response for that picture. On average, there was $88 \%$ agreement between the word used in the Federmeier and Kutas stimuli and the naming response to the corresponding line drawing used in this experiment.

The first sentence of each pair established the expectation for the target item and its category. In contrast, the second sentence if presented alone could be completed plausibly by any of the three possible targets. Targets were objects from 66 categories (two from each). Categories were chosen to be those at the lowest level of inclusion for which the average undergraduate student could be expected to readily differentiate several exemplars. For approximately half the categories used, this level was basic as determined by Rosch et al. [59] or by analogy. Other categories were based at the next highest level (a superordinate of the basic-level) because it was unclear that the average participant could clearly and consistently differentiate below this level. To help control for the plausibility of the two violation types, between category targets for each sentence pair were chosen from a related category that shared key features (e.g. animacy, size, general function) with that from which the expected exemplar and within category violation were derived.

Target items were rotated across the stimulus set such that each item appeared six times, once as each kind of ending (3) in each visual field (2). Thus, across the experiment all conditions were perfectly controlled for both lexical and visual properties of the target, and context sentences in each ending type condition also were perfectly controlled for length and grammatical complexity. Picture stimuli subtended $4.3^{\circ}$ of horizontal and vertical visual angle. The experimental sentences were divided into six lists of 132 sentences each; each participant viewed only one list. Sentence contexts and items were used only once per list, and each list consisted of 44 of each type of target (expected exemplars, within category violations, between category violations), half presented in the right and half in the left visual field ( 22 per field). To balance the number of plausible and implausible sentences read by each participant, the same 44 plausible filler 
sentence pairs were added to each list, half in each visual field.

\subsection{Cloze procedure and expectancy ratings}

Cloze probabilities were obtained for the 132 sentence pair contexts (sentence pairs missing the final item of the second sentence). These were divided into two lists, such that the two sentence contexts presumed to be predictive of items coming from the same category did not both appear on the same list. Student volunteers were asked to complete each sentence pair with "the first word that comes to mind". List 1 was completed by 56 students and list 2 was completed by a different set of 59 students. A subset of the original stimuli were rewritten and clozed separately by a third group of 55 students. Cloze probability for a given word in a given context was calculated as the proportion of individuals choosing to complete that particular context with that particular word. Expected exemplars were always the item with the highest cloze probability for a given context (mean $=0.74$ ). All violations had cloze probabilities of less than 0.05 , with a mean cloze probability of 0.004 for the within category violations and 0.001 for the between category violations. To ensure that within and between category violations (which, with rare exceptions, were not generated in the cloze task) were in fact considered implausible completions for the sentence contexts, plausibility ratings of all items in their sentence contexts were also obtained from a different group of student volunteers. These ratings confirmed that, in addition to not being produced in the sentence contexts, both violation types were regarded as surprising/difficult to integrate when placed into them; see Federmeier and Kutas [22] for details and analyses.

\subsection{Participants}

Eighteen UCSD undergraduate volunteers ( 8 women, 18-28 years of age, mean age 20) participated in the experiment for course credit or cash. All were right-handed (as assessed by the Edinburgh Inventory [50]) monolingual English speakers with normal vision and no history of reading difficulties or neurological/psychiatric disorders; none had a left-handed immediate relative. Three participants were randomly assigned to each of the six stimulus lists.

\subsection{Experimental procedure}

Volunteers were tested in a single experimental session. To facilitate picture identification and thereby reduce eye movements to laterally presented items, participants were pre-exposed to all the line drawings prior to EEG recording (note that this "familiar picture" condition produced results most similar to word processing with central presentation of these stimuli [23]). Line drawings were presented centrally on a computer monitor in the same size and orientation as used in the experimental trials. Participants pushed a button to move through the set of pictures, which were presented in random order, and were instructed to write down what they thought each picture represented and to rate the "quality" of the line drawing as a representation of that object on a seven point scale, from "I have difficulty determining what this line drawing is supposed to represent" (1) to "This line drawing is one of the best possible representations I can imagine for this item" (7). The average quality rating across items was 5.3 (range 2.8-6.5), indicating that the participants found the line drawings to be reasonably good representations of the target concepts.

EEG recording was conducted in a soundproof, electrically-shielded chamber. Participants were seated in a comfortable chair $40 \mathrm{in}$. in front of a monitor and instructed to read the stimulus sentences for comprehension. They also were informed that they would be given a recognition memory test over the stimuli at the conclusion of recording. The session began with a short practice run.

Each trial began with the first sentence of a pair appearing in full on a CRT. Volunteers read this sentence at their own pace and pushed a button to view the second sentence. Presentation of the second sentence was preceded by a series of crosses to orient the volunteer toward the center of the screen. The second sentence was then presented one word at a time horizontally for a duration of $200 \mathrm{~ms}$ with a stimulus onset asynchrony of $500 \mathrm{~ms}$; sentence-final pictures were also presented for a duration of $200 \mathrm{~ms}$. Sentence context words were presented in the center of the screen while sentence-final pictures were presented pseudo-randomly ${ }^{2}$ in the left or right visual hemifield with inner edge $2^{\circ}$ of visual angle from fixation. A central fixation point remained visible throughout the trial, positioned $1 / 2^{\circ}$ below the bottom-most edge of the centrally presented words. Volunteers were asked not to blink or move their eyes during the second sentence. The target picture was followed by a blank screen for $3000 \mathrm{~ms}$, after which the next sentence appeared automatically. Volunteers were given a short break after every 17 pairs of sentences.

At the conclusion of the recording session, participants were given a recognition memory test consisting of 50 sets of sentence pairs: 10 new, 20 unchanged experimental pairs (of which 10 ended with expected exemplars, 5 ended with within category violations, and 5 ended with between category violations), and 20 modified sentence pairs in which the final item had been changed from that originally viewed by the volunteer (10 in which violations had been changed to expected exemplars and 10 in which expected exemplars had been changed to violations). Pictures were not used in the recognition memory test; instead, each sentence-final item was the word corresponding to the picture's label.

\footnotetext{
${ }^{2}$ Equal numbers of each ending type (expected exemplar, within category violation, and between category violation) were shown to each hemisphere in an experimental session. Order of presentation was randomized with the stipulation that not more than three stimuli in a row be presented to the same hemifield.
} 
Volunteers were instructed to classify the sentences as new, old, or similar (changed).

\subsection{EEG recording parameters}

The electroencephalogram (EEG) was recorded from 26 tin electrodes embedded in an electro-cap, referenced to the left mastoid. These sites included midline prefrontal (MiPf), left and right medial prefrontal (LMPf and RMPf), left and right lateral prefrontal (LLPf and RLPf), left and right medial frontal (LMFr and RMFr), left and right mediolateral frontal (LDFr and RDFr), left and right lateral frontal (LLFr and RLFr), midline central (MiCe), left and right medial central (LMCe and RMCe), left and right mediolateral central (LDCe and RDCe), midline parietal (MiPa), left and right mediolateral parietal (LDPa and RDPa), left and right lateral temporal (LLTe and RLTe), midline occipital (MiOc), left and right medial occipital (LMOc and RMOc), and left and right lateral occipital (LLOc and RLOc). Blinks and eye movements were monitored via electrodes placed on the outer canthus (left electrode serving as reference) and infraorbital ridge of each eye (referenced to the left mastoid). Electrode impedances were kept below $5 \mathrm{k} \Omega$. EEG was processed through Grass amplifiers set at a bandpass of $0.01-100 \mathrm{~Hz}$. EEG was continuously digitized at $250 \mathrm{~Hz}$ and stored on hard disk for later analysis.

\subsection{Data analysis}

Data was re-referenced off-line to the algebraic sum of the left and right mastoids. Trials contaminated by eye movements, ${ }^{3}$ blinks, excessive muscle activity, or amplifier blocking were rejected off-line before averaging; approximately $11 \%$ of trials in each hemifield were lost due to such artifacts (maximum rejection rate $30 \%$ ). ERPs were computed for epochs extending from $100 \mathrm{~ms}$ before stimulus onset to $920 \mathrm{~ms}$ after stimulus onset. Averages of artifact-free ERP trials were calculated for each type of target picture (expected exemplars, within category violations, between category violations) in each hemifield (right and left) after subtraction of the $100 \mathrm{~ms}$ pre-stimulus baseline.

As in the central picture study [23], we examine the effects in four time windows: 75-175 ms (N1), 150-250 ms (P2), 250-350 ms (N300), and 350-500 ms (N400). Using the same strategy for analysis as that used in the lateralized word study [21], we first examine whether the two hemispheres differ in their response to the congruent completions and then examine the N400 effect (difference between incongruent and congruent responses) to the two violation types. Latency and mean amplitude measures were subjected to a

\footnotetext{
${ }^{3}$ Peak to peak amplitude measurements were used to identify and reject trials containing saccades during averaging. The threshold for rejection was set on a subject by subject basis, using visual inspection of all target trials to ensure that those containing appreciable saccade-related activity would be eliminated.
}

repeated measures omnibus analysis of variance (ANOVA). For latency measures, repeated measures included two levels of hemifield (right versus left) and 26 levels of electrode. In order to assess distributional effects for mean amplitude measures the electrode factor was broken down into two levels of hemisphere (left versus right), ${ }^{4}$ two levels of laterality (lateral versus medial), and four levels of anterior/posterior (prefrontal versus frontal versus parietal versus occipital). ${ }^{5}$ Repeated measures analyses of incongruent items included two levels of ending type (within category violation versus between category violation) in addition to the measures already described. Note that $P$-values are reported after epsilon correction (Huynh-Felt) for repeated measures with greater than 1 degree of freedom.

\section{Results}

\subsection{Behavior}

Participants correctly classified an average of $85 \%$ (range $68-100 \%$ ) of the items on the recognition memory test. The most common type of error was a misclassification of "similar" sentences (those in which the final word did not match the picture seen in that sentence context) as "old" (35\% of all errors), followed by a misclassification of "old" sentences (those in which the final word did match the picture seen in that context) as "similar" (28\% of all errors). The remainder of the errors primarily consisted of participants classifying "old" or "similar" sentences as "new" (14 and $21 \%$ of all errors, respectively). There were two errors in which "new" sentences were classified as "similar"; no participant ever incorrectly said "old" to a "new" sentence. In general, the results indicate that participants were attending to the experimental stimuli during the recording session and were successful at identifying and remembering line drawings presented in the visual periphery.

\subsection{ERPs}

Fig. 1 shows grand average ERPs to sentence-final pictures in each visual field. In all conditions and hemifields, early components include, at frontal sites, a negativity peaking around $125 \mathrm{~ms}(\mathrm{~N} 1)$ and a positivity peaking around $200 \mathrm{~ms}$ (P2), and at posterior sites, a positivity peaking around $100 \mathrm{~ms}(\mathrm{P} 1)$, a negativity peaking around $150 \mathrm{~ms}$ (N1), and a positivity peaking around $200 \mathrm{~ms}$ (P2). As expected, these effects (posterior ones in particular) are strongly lateralized, being most prominent over electrode sites contralateral to the visual half-field of presentation.

\footnotetext{
${ }^{4}$ To avoid confusion, in all our analyses we use the term "hemifield" to refer to the location of the stimulus on the screen and the term "hemisphere" to refer to the location of electrodes on the head.

${ }^{5}$ Left lateral sites (from front to back): LLPf, LLFr, LLTe, LLOc; left medial sites: LMPf, LMFr, LMCe, LMOc; right medial sites: RMPf, RMFr, RMCe, RMOc; right lateral sites: RLPf, RLFr, RLTe, RLOc.
} 
RIGHT VISUAL FIELD

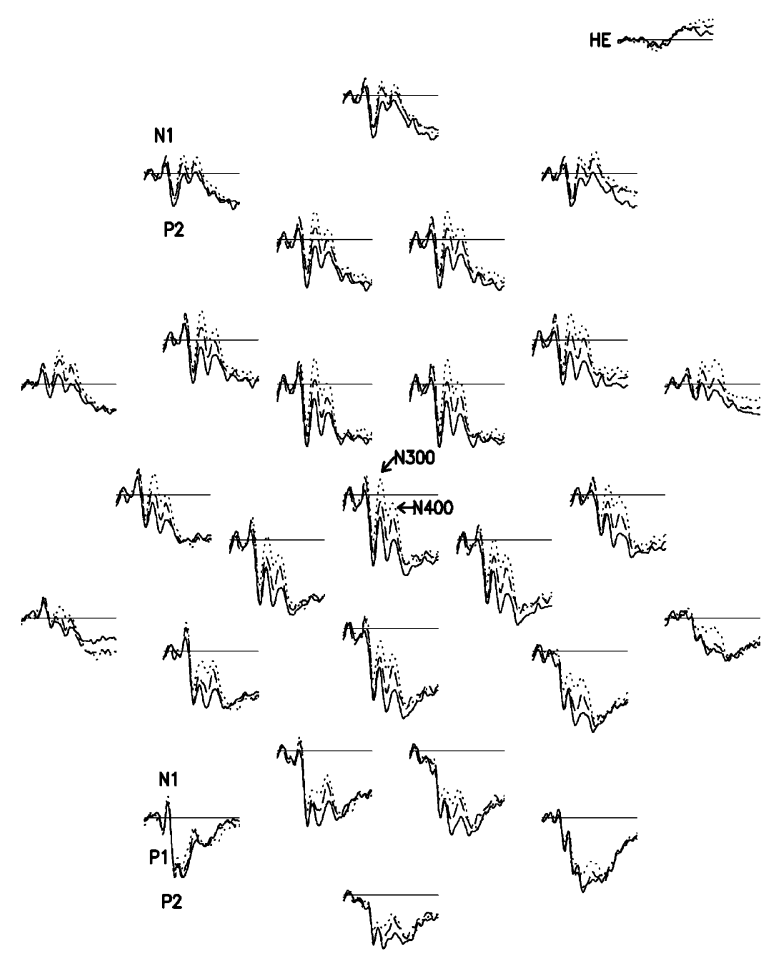

LEFT VISUAL FIELD

HE maxing

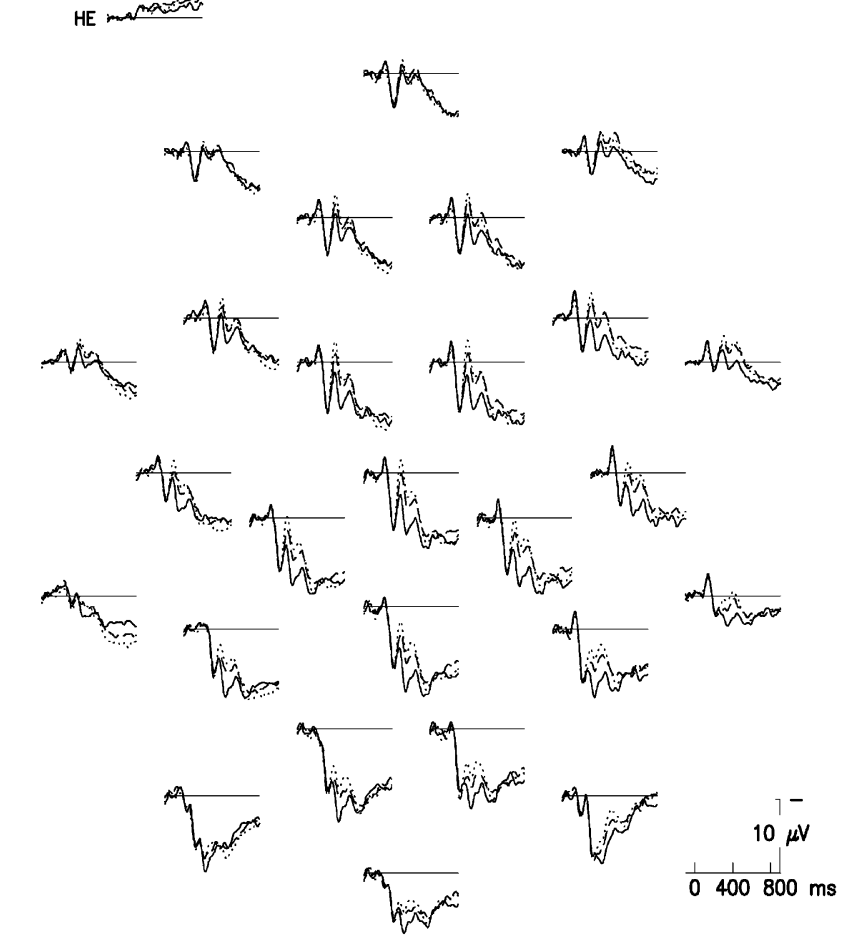

- Expected Exemplars ---- Within Category Violations

Between Category Violations

Fig. 1. Grand average $(N=18)$ ERP waveforms at all 26 scalp electrode sites. ERPs elicited by pictures during right visual field (left hemisphere) presentation are shown on the left and ERPs elicited by pictures during left visual field (right hemisphere) presentation are shown on the right. Negative is plotted up and major components are labeled. A negativity peaking around $300 \mathrm{~ms}$ (N300) differentiated the three ending types but did not vary with visual field of presentation while a later negativity peaking around $425 \mathrm{~ms}(\mathrm{~N} 400)$ varied as a function of both ending type and visual field of presentation. Recordings from the horizontal eye channel (right horizontal eye referenced to the left horizontal eye) are also shown for presentations in each visual field; these recordings reveal that ending type differences are not confounded with eye movement differences.

In all conditions, these early components are followed by broadly-distributed negativities, peaking at approximately 300 and $425 \mathrm{~ms}$, which vary in amplitude as a function of hemifield of presentation and ending type. The negativities are followed by an extended late positivity of similar amplitude across conditions.

\subsubsection{Response to expected exemplars}

3.2.1.1. N1/P2 effects. Mean amplitude measures were taken in the $75-175 \mathrm{~ms}$ (N1) time window and the 150-200 ms (P2) time window. In the N1 time window there was a trend toward larger overall amplitudes for left as opposed to right visual field presentations that failed to reach significance $(F(1,17)=2.51 ; P=0.13)$. Distributional effects after normalization revealed a significant hemifield by hemisphere interaction $(F(1,17)=8.79$; $P<0.01)$, reflecting the fact that N1 responses were largest over scalp sites contralateral to the visual field of presentation. There was a significant effect of hemifield in the P2 time window $(F(1,17)=5.07 ; P<0.05)$, with more positive responses for right than for left visual field presen- tations. This effect did not interact with any distributional variable.

3.2.1.2. N300/N400 effects. Latency of the largest negative peak was measured in the N300 (250-350 ms) and N400 (350-500 ms) time windows for each hemifield condition in each subject. Peaks were found at 294 and $425 \mathrm{~ms}$ in the right visual field and at 290 and $428 \mathrm{~ms}$ in the left visual field. These latencies did not differ as a function of hemifield in either time window $((F(1,17)=1.95 ; P=\mathrm{ns})$ and $(F(1,17)=0.13 ; P=\mathrm{ns})$, respectively $)$.

Mean amplitude measures were then taken in the same two time windows. Mean amplitudes of the N300 and N400 responses were 3.96 and $4.52 \mathrm{mV}$ in the right visual field and 3.65 and $4.34 \mathrm{mV}$ in the left visual field; there was no main effect of hemifield in either the $\mathrm{N} 300(F(1,17)=$ $0.37 ; P=\mathrm{ns})$ or the $\mathrm{N} 400(F(1,17)=0.15 ; P=\mathrm{ns})$ time window. Distributional effects after normalization [44] included, in the N300 time window, a significant hemifield by hemisphere by laterality interaction $(F(1,17)=4.68 ; P=$ $0.04)$ modulated by a significant hemifield by hemisphere by laterality by anteriority interaction $(F(3,51)=4.39$; 
$P=0.02)$. In the N400 time window, effects included a significant hemifield by hemisphere interaction $(F(1,17)=$ 17.16; $P<0.001)$ modulated by a significant hemifield by hemisphere by laterality interaction $(F(1,17)=17.83$; $P<0.001)$ and a hemifield by hemisphere by anteriority interaction $(F(3,51)=26.46 ; P<0.001)$; a trend toward a four-way interaction was also observed $(F(3,51)=2.65$;
$P=0.09$ ). All other distributional effects were not significant.

This difference in the distribution of the electrical response to items presented in the right versus left visual half-field can be seen in Fig. 2. It begins in the early time window, is fully developed in the late time window, and continues to the end of the epoch. In all these time

EXPECTED EXEMPLARS

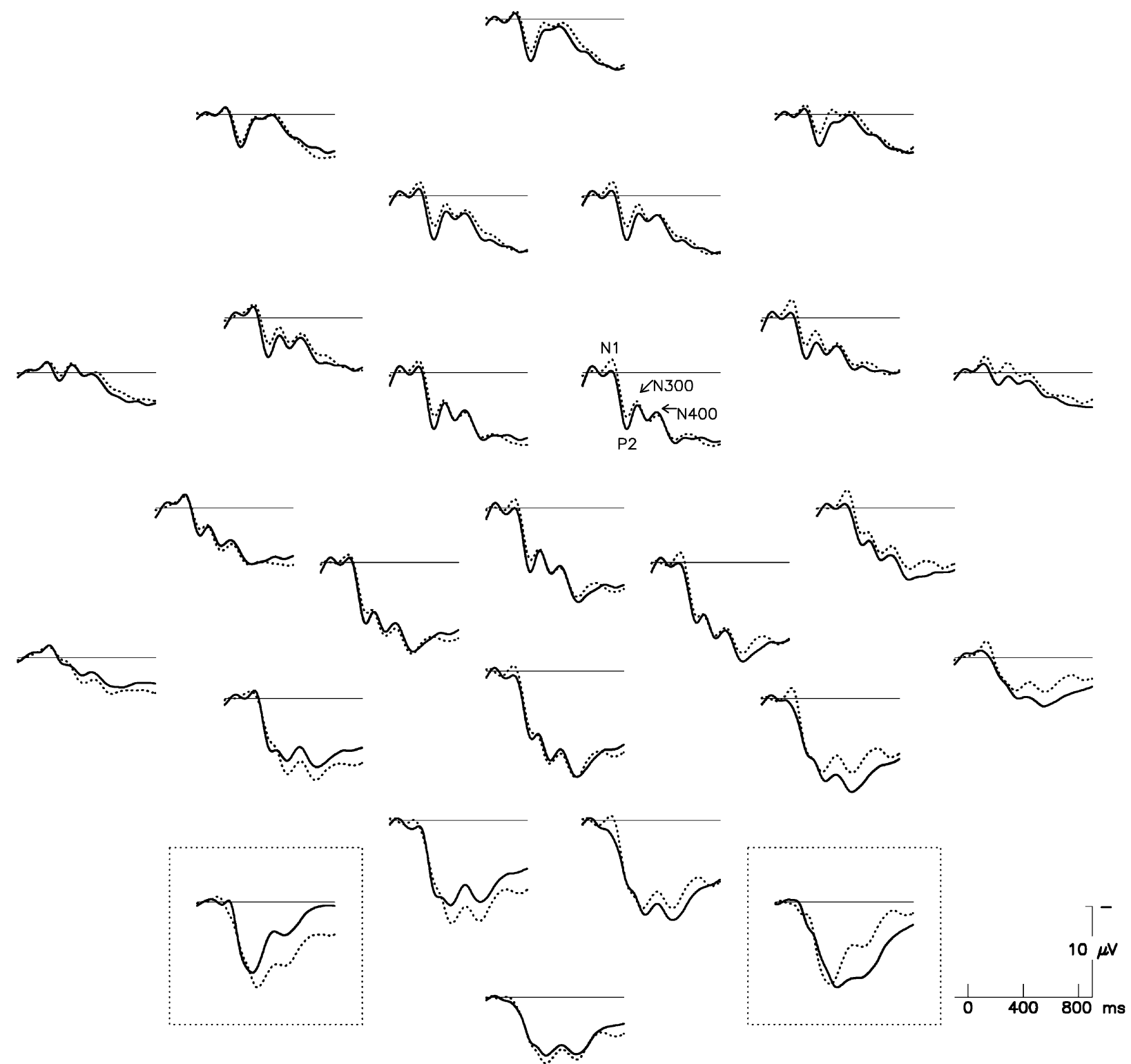

Right Visual Field/Left Hemisphere

Left Visual Field/Right Hemisphere

Fig. 2. Response to expected exemplars as a function of visual field of presentation. Over most of the scalp, responses to expected pictures presented to the right (solid) and left (dotted) visual fields were quantitatively and qualitatively similar. Over the back of the head, however, more positive responses were observed over sites ipsilateral to the visual field of presentation, as can be seen by comparing the two boxed electrodes. 
windows, there is a larger positivity over right than left medial posterior sites to stimuli presented in the right visual field and a larger positivity over left than right medial posterior sites to stimuli presented in the left visual field. This pattern was previously observed to sentence-final words in the same sentence contexts [21] and in response to lateralized single words [48].

3.2.1.3. Summary. N1 effects were lateralized to scalp sites contralateral to the visual field of presentation and slightly smaller for right visual field presentations. In addition, $\mathrm{P} 2$ responses to expected exemplars were larger (more positive) with right than with left visual field presentations.

Overall, responses to contextually expected targets in the two visual fields were equivalent in their latency and their mean amplitude for both the N300 and the N400 time windows. The only significant effect was a distributional interaction in which stimuli presented to a single visual field are associated with larger positivities at ipsilateral (as compared to contralateral) medial posterior electrode sites.

\subsubsection{Response to violations}

3.2.2.1. N1/P2 effects. Effects of hemifield and violation type were examined in the $75-175 \mathrm{~ms}(\mathrm{~N} 1)$ and the 150-200 ms (P2) time windows. While there were no significant effects in the N1 time window, there was again a significant effect of hemifield in the P2 time window $(F(1,17)=9.29 ; P<0.01)$ which did not interact with any distributional variables. In general, $\mathrm{P} 2$ responses varied with ending type with right visual field presentations (larger for within than for between category violations) $(F(2,34)=6.32 ; P<0.01)$, but were of equal magnitude to both violation types with left visual field presentation $(F(2,34)=0.16 ; P=\mathrm{ns})$. This difference arises because P2 responses to between category violations were less positive with right than with left visual field presentation $(F(1,17)=6.20 ; P<0.05)$. The response to within category violations was not significantly different as a function of visual field $(F(1,17)=0.33 ; P=\mathrm{ns})$.

3.2.2.2. N300/N400 effects. We measured the latency of the largest negative peak in the N300 and N400 time windows for each violation type. Table 2 gives these latencies as a function of hemifield and ending type for both time windows (with standard errors in parentheses).

Peak latencies did not vary as a function of either hemifield $(\mathrm{N} 300$ time window $(F(1,17)=1.59 ; P=\mathrm{ns}) ; \mathrm{N} 400$ time window $(F(1,17)=0.39 ; P=\mathrm{ns})$ or of ending type (N300 time window $(F(1,17)=0.41 ; P=\mathrm{ns})$; N400 time window $(F(1,17)=1.29 ; P=\mathrm{ns})$ and the two variables did not interact.

To examine N300/N400 amplitude effects across hemifield and violation type, we first computed the ERP difference between each violation type and the expected exemplar from the same hemifield condition. Since in the time windows of interest there were no latency or amplitude differences in the response to expected exemplars as a function of hemifield, using difference waves is equivalent to looking at the raw N400 responses. Using difference waves, however, has the advantage of canceling out standing distributional differences between the hemifields (like that discussed in the previous section) and of allowing more straightforward comparisons between the response to pictures in this experiment and the response to words in Federmeier and Kutas [21]. Fig. 3 shows these difference waves at all channels. Mean amplitude measures for each difference wave (RVF within category violation minus RVF expected exemplar; RVF between category violation minus RVF expected exemplar; LVF within category violation minus LVF expected exemplar; LVF between category violation minus LVF expected exemplar) were then taken in the same two time windows. Table 3 gives these mean amplitude differences as a function of hemifield and ending type for both time windows (standard errors in parentheses).

In the N300 time window, there was a significant effect of ending type $(F(1,17)=15.00 ; P=0.001)$ but no effect of hemifield $(F(1,17)=0.78 ; P=$ ns $)$ and no hemifield by ending type interaction $(F(1,17)=1.92 ; P=\mathrm{ns})$. Planned comparisons revealed that between category violations were more negative than within category violations in both visual fields, though the difference was only marginally significant for the left visual field $\operatorname{RVF}(F(1,17)=16.03 ; P=$ $0.001) ; \operatorname{LVF}(F(1,17)=3.03 ; P=0.10))$. The response to within category violations did not differ as a function of hemifield in this early time window $(F(1,17)=0.27$; $P=\mathrm{ns}$ ), while the response to between category violations showed a non-significant trend for greater negativity to stimuli presented in the right visual field $(F(1,17)=2.75 ; P=$ 0.11 ).

In the $\mathrm{N} 400$ time window, there was again a significant effect of ending type $(F(1,17)=7.11 ; P=0.02)$, in this case modulated by a marginal hemifield by ending type interaction $(F(1,17)=3.32 ; P=0.09)$. There was no main effect of hemifield $(F(1,17)=0.65 ; P=\mathrm{ns})$. Planned

Table 2

Peak latencies (ms) across all channels

\begin{tabular}{llllll}
\hline & \multicolumn{2}{l}{ N300 time window $(250-350 \mathrm{~ms})$} & & \multicolumn{2}{l}{ N400 time window (350-500 ms) } \\
\cline { 2 - 3 } \cline { 5 - 6 } & RVF & LVF & & RVF & LVF \\
\hline Within category violation & $296(1.4)$ & $301(1.3)$ & & $419(2.0)$ & $422(2.2)$ \\
Between category violation & $299(1.3)$ & $301(1.2)$ & $420(1.9)$ & $411(2.2)$ \\
\hline
\end{tabular}




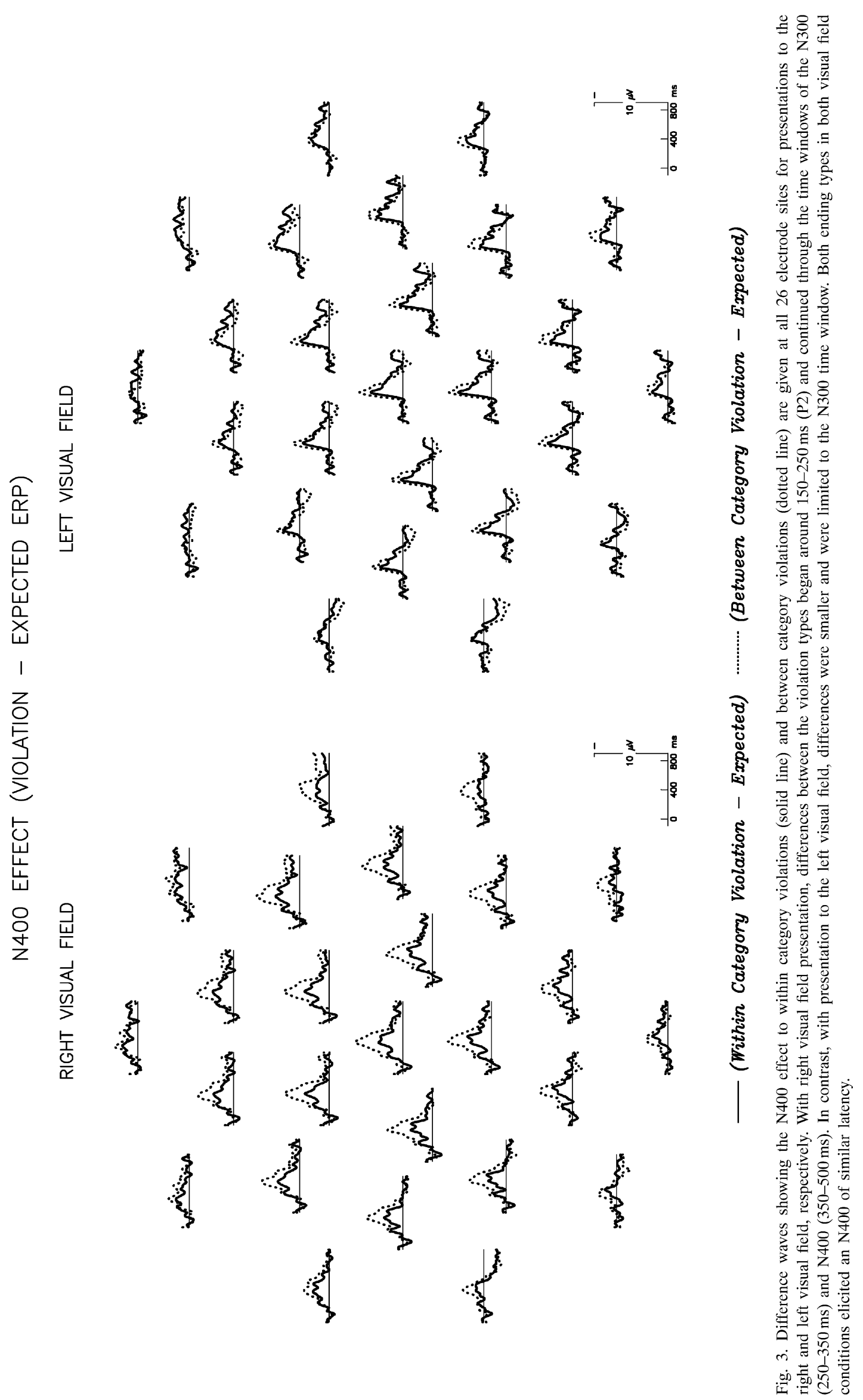


Table 3

Mean amplitudes $(\mathrm{mV})$ across all channels

\begin{tabular}{|c|c|c|c|c|}
\hline & \multicolumn{2}{|c|}{ N300 time window $(250-350 \mathrm{~ms})$} & \multicolumn{2}{|c|}{ N400 time window $(350-500 \mathrm{~ms})$} \\
\hline & RVF & LVF & RVF & LVF \\
\hline Within category violation & $-1.53(0.20)$ & $-1.50(0.20)$ & $-1.52(0.14)$ & $-1.80(0.18)$ \\
\hline Between category violation & $-3.37(0.24)$ & $-2.40(0.23)$ & $-3.26(0.18)$ & $-2.15(0.23)$ \\
\hline
\end{tabular}

comparisons revealed that between category violations were more negative than within category violations in the right visual field $(F(1,17)=13.61 ; P<0.01)$ but did not differ in the left visual field $(F(1,17)=0.32 ; P=\mathrm{ns})$. The response to within category violations again did not differ as a function of hemifield, but the response to between category violations was significantly larger for stimuli presented in the right as compared with the left visual field $(F(1,17)=$ 4.28; $P=0.05$ ).

Distributional effects of hemifield after normalization were similar in both time windows. There was a hemifield by hemisphere by laterality effect in the N300 time window $(F(1,17)=4.77 ; P=0.04)$ which was marginal in the N400 time window $(F(1,17)=3.06 ; P=0.10)$. Responses were generally more negative over medial than over lateral sites; however, relative to all other hemifield by hemisphere combinations, the difference between medial and lateral electrode sites was steeper over left hemisphere electrode sites for stimuli presented to the left visual field. All other interactions with hemifield were not significant.

In the N400 time window, there was also a significant ending type by hemisphere by laterality interaction $(F(1,17)=$ 5.23; $P=0.04)$ and a marginal ending type by hemisphere by anteriority interaction $(F(3,51)=2.17 ; P=0.10)$. The difference in response size between within and between category violations was smaller over left, lateral sites than over all other sites (consistent with the fact that $\mathrm{N} 400$ responses tend to be bigger medially and over right hemisphere sites). In general, N400 responses are larger over right than left hemisphere sites and larger over central than over prefrontal or occipital sites. However, for between category violations, the difference between right and left hemisphere sites is especially large over central electrode sites relative to more frontal or posterior sites, while the right-left difference is similar as a function of anteriority for within category violations.

3.2.2.3. Summary. In summary, while $\mathrm{N} 1$ amplitudes did not vary with hemifield or violation type, $\mathrm{P} 2$ amplitudes did. With presentation to the left visual field, P2 responses were the same across ending types. In contrast, with right visual field presentations, $\mathrm{P} 2$ responses were smaller (less positive) to between category violations. Responses to within category violations did not differ as a function of hemifield, while responses to between category violations were smaller with presentation to the right than to the left visual field.
While N300 and N400 peak latencies did not differ with either hemifield or violation type, the amplitude of these components was affected by both variables. When pictures were presented in the right visual field, larger negativites were observed to between than to within category violations in both the N300 and the N400 time windows. In contrast, when these same stimuli were presented in the left visual field a difference between the violations types was observed only in the N300. Fig. 4 shows this contrast, which, in the N400 time window, is similar to that observed for sentence-final words [21]. Responses to within category violations did not differ as a function of hemifield in either time window. The response to between category violations, in contrast, was significantly more negative in the N400 time window when these were presented to the right as opposed to left visual field (with a non-significant trend in this direction for the N300 time window). The different pattern observed in the two hemifields thus seems to be driven by differences in the response to between category violation pictures.

\subsubsection{Comparison of the N300 and N400 effects}

To further characterize the two negativities observed between 250 and $500 \mathrm{~ms}$ in this experiment, we performed a distributional analysis comparing the N300 and N400 effects. Mean amplitude differences (violations minus expected exemplars, collapsed across violation type) were subjected to ANOVA on five repeated measures: two levels of effect (N300, measured from 250 to $350 \mathrm{~ms}$ versus $\mathrm{N} 400$, measured from 350 to $500 \mathrm{~ms}$ ), two levels of hemifield (right versus left), two levels of hemisphere (left versus right), two levels of laterality (lateral versus medial), and four levels of anterior/posterior (prefrontal versus frontal versus parietal versus occipital).

We found an effect by hemifield interaction $(F(1,17)=$ $6.62 ; P=0.02)$ and an effect by anteriority interaction $(F(3,51)=4.05 ; P=0.03)$, which was modulated by a marginally significant effect by laterality by anteriority interaction $(F(3,51)=3.06 ; P=0.06)$. Whereas the $\mathrm{N} 400$ effect is more negative over right than left hemisphere sites, the N300 effect is bilaterally symmetric. The two effects are similar in amplitude over frontal and parietal sites, but the N400 is larger over occipital sites whereas N300 is larger over prefrontal sites. This difference between the effects at occipital sites tended to be more pronounced over lateral electrode locations, while the difference over frontal sites tended to be largest over medial electrode locations. No interactions with hemifield were observed, indicating that 

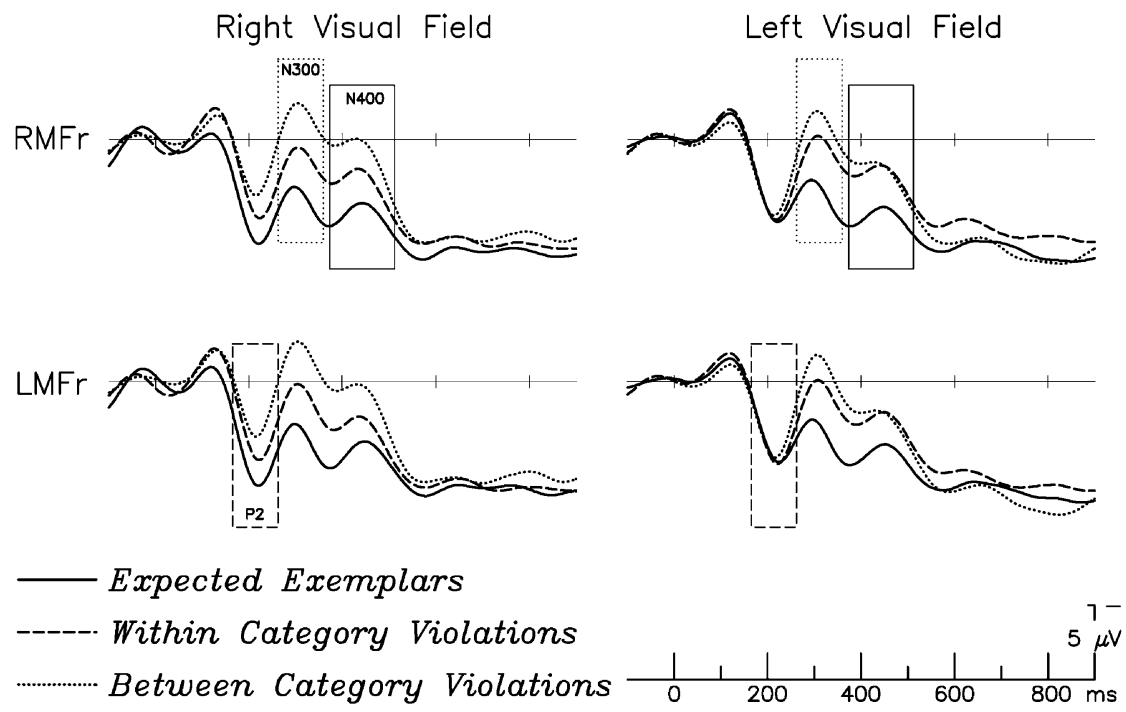

Fig. 4. The ending type effect shown at right and left medial frontal sites where effects tended to be largest. For right visual field (left hemisphere) presentations (left side of the figure), the three ending types begin to be differentiated in the P2 (150-250 ms) time window (dashed box), while effects with left visual field (right hemisphere) presentations do not begin until the time window of the N300 (250-350 ms) (dotted box). In both visual fields, N300 responses are smallest (least negative) to expected pictures and largest (most negative) to unexpected pictures from an unexpected category (between category violations). In the right visual field, a similar three-way split can also be observed in the amplitude of the N400 response (350-500 ms) (solid box). For left visual field, in contrast, N400s of similar amplitude were elicited by within and between category violations, both of which were more negative than the response to expected exemplars. Responses to both violation types in the right hemisphere were similar in amplitude to responses to within category violations in the left hemisphere.
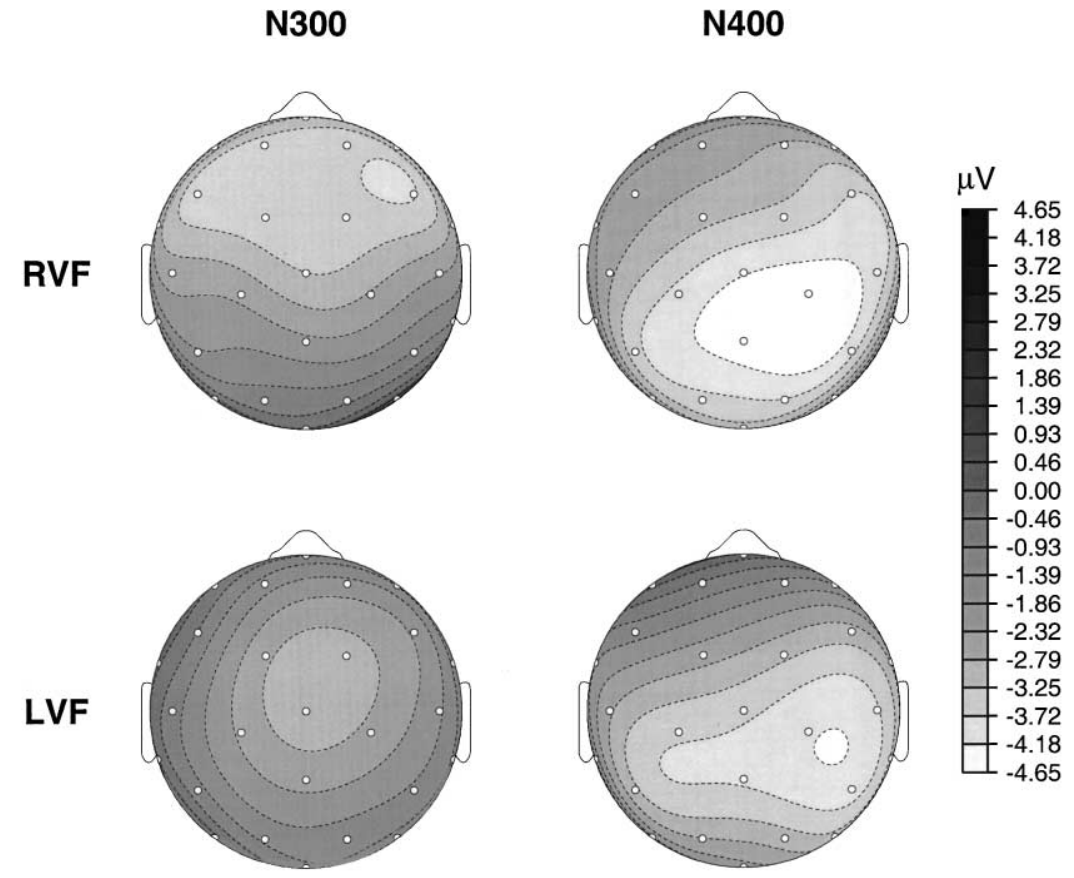

Fig. 5. Voltage maps showing the distribution of the N300 (250-350 ms) and N400 (350-500 ms) effects with right and left visual field presentation. Effects are collapsed across violation type. For presentation to both visual fields, the N400 response (right side of figure) was largest over central-posterior sites and bigger over right than left scalp locations. In contrast, the N300 response (left side of figure) was more bilaterally symmetric and frontal in its distribution. 
the distribution of both effects was similar across visual field of presentation.

Overall, then, the distribution of the N400 effect was typical for word by word sentential reading: largest central-posteriorly and bigger over the right than the left side of the head ${ }^{6}$ (e.g. [39]). In contrast, the distribution of the N300 was more frontal (as was also observed by McPherson and Holcomb [46]) and did not show a lateral bias. Fig. 5 gives voltage maps showing these distributions.

\section{Discussion}

We discovered previously that the left and right hemispheres differ in how they process words in the context of sentence pairs [21]. We also uncovered differences in the semantic feature information extracted from words and pictures when these are presented in central vision [22,23]. In this experiment, we sought to compare picture processing in the two hemispheres and to thereby examine whether, and if so how, modality differences might interact with hemispheric processing differences. The issue of how the two hemispheres process semantic information presented as a picture as opposed to a word has been difficult to answer heretofore because no prior study has used the same types of stimuli and task conditions to compare them. Here, individuals read for comprehension as we measured the electrophysiological response to lateralized line drawings representing the same concepts that we previously examined using words; to our knowledge, this is the first study to use electrophysiological measures to examine hemispheric differences in picture processing. We compared responses to contextually expected items and also to contextually unexpected items that were or were not semantically related to the expected item when these target types were presented in the left versus in the right visual half-field.

When we examined lateralized word processing in these same sentence pair contexts [21], we found that N400 responses reflected the congruency of the item with the preceding context irrespective of visual field. In fact, when compared directly, responses to expected words presented in the two visual fields did not differ in timing or amplitude (with symmetrical distributional differences). Behavioral work has suggested that the two hemispheres are even more similar in their semantic processing of isolated pictures than of isolated words (e.g. [4,16,17,41]). Furthermore, Bie-

\footnotetext{
${ }^{6}$ Note that scalp distribution effects are independent of the laterality effects as a function of hemifield of presentation that are of primary theoretical interest here. Regardless of presentation location, N400 effects tend to be seen most prominently over medial central scalp locations and are generally slightly larger over right than left hemisphere sites. This distributional information, however, cannot be used to make direct inferences about the lateralization of the neural activity responsible for the N400. We do not make inferences based on scalp distribution here and, indeed, do not find joint interactions of hemifield and ending type with any distributional variables.
}

derman and Cooper [4] have shown that picture processing in both hemispheres can be facilitated by the prior presentation of a (closely) semantically-related picture. However, we do not know of any prior study that has actually examined the influence of a verbal context on picture processing in the two hemispheres. Based on the pattern observed for words, we predicted an N400 reduction to expected pictures in both visual fields. As predicted, we found that, regardless of which hemisphere initially receives the information, responses to contextually expected (as compared with unexpected) pictures are characterized by increased positivity between 350 and $500 \mathrm{~ms}$, showing that both hemispheres differentiate items that semantically fit the verbal context from those that do not. Indeed, when compared directly, the ERP responses to expected items presented in the left and right visual half-fields do not differ in amplitude or latency and are identical in distribution over most of the scalp (with mirror image effects as a function of hemifield seen over medial, posterior electrodes). This is the same pattern as we observed previously for the word processing [21].

It has been proposed that the two hemispheres actually extract different types of information from a verbal context. For example, based on the results of behavioral experiments using words, Faust and coworkers suggest that context effects in the left hemisphere reflect a sensitivity to message-level meaning, whereas context effects in the right hemisphere result instead from lexical associative relationships disconnected from message-level meaning [19,20]. Alternatively, we have suggested that the left hemisphere, but not the right, uses context predictively, i.e. it preactivates some of the semantic features of upcoming items [21]. Irrespective of how the difference is characterized, however, the similarity in the ERP response to expected items in the two visual half-fields implies that if the two hemispheres do use different strategies when analyzing a verbal context, these strategies can nevertheless facilitate later processing in a very similar manner. And this is true whether the target concept is represented by a word or by a picture.

While we found that the response to lateralized words was similar in the two visual fields when these were expected, we found a qualitatively different pattern of N400 responses to the two types of unexpected words for right versus left initiated processing [21]. Responses with right hemisphere initiated processing patterned directly with the plausibility of these items in the sentence pair contexts, with no difference in N400 amplitudes to within as compared with between category violations. In contrast, with left hemisphere initiated processing, responses to within category violations were smaller than responses to between category violations. Left hemisphere processing thus showed a sensitivity to the degree of feature overlap between the violation and the word that was only predicted in the context, suggesting that features of that predicted word had become activated prior to its occurrence. Assuming that these word processing differences arise because of general differences in how the two hemispheres use context information in real time, we would 
predict the same pattern of N400 responses to lateralized pictures in this study.

Here, again, we find the predicted pattern of responses. When first presented to the left hemisphere (right visual field), between category violations elicit larger N400s than do within category violations. In contrast, when first presented to the right hemisphere (left visual field) the two violation types elicit equivalent N400 responses. In other words, semantic integration by the left hemisphere reflects the influence of both contextual fit and semantic memory organization while semantic integration by the right hemisphere seems to be sensitive primarily to contextual fit (that only the left hemisphere is sensitive to categorical relationships between pictures was also the conclusion reached by Hines et al. [31]). Thus, independent of modality of input, neural processing of items initially presented to the left visual field (right hemisphere) seem to be driven primarily by the plausibility of those items in the current sentence context. We have termed this an "integrative" pattern of contextual processing: items are facilitated when a sufficient number of their semantic features cohere with those of the context. In contrast, contextual processing by the left hemisphere seems to involve a predictive component. The relationship between an item that is presented and an item that is only expected in a context can affect processing only if one assumes that exposure to the context preactivated (i.e. predicted) semantic features associated with that expected item. The N400s associated with left hemisphere processing of both pictures and words reflect the fit of these unexpected target items, not to the context itself, but rather to the item most expected in a context, indicating that the left hemisphere is making predictions.

However, while we find a qualitatively similar hemispheric difference for picture and for word processing, there are modality-based differences as well. For words, the difference between the hemispheres seemed to be driven by differences in the response to within category violations. That is, while there were no differences in the response to between category violations as a function of visual field, N400 responses to within category violations were reduced during left as compared with right hemisphere initiated processing [22]. This is not what we observe here for picture processing in the two hemispheres; instead, the difference seems to be driven by the responses to between category violations. We find no difference in the response to within category violations as a function of visual field, but an increased N400 response to between category violations in left hemisphere as compared with right hemisphere initiated processing. These differences mirror the modality differences that are observed in central vision as a function of sentential constraint [23]. ${ }^{7}$ Increased constraint decreases

\footnotetext{
${ }^{7}$ This modality difference in the central picture study was observed for both the kind of familiar (pre-exposed) pictures used here and for unfamiliar (non-pre-exposed) pictures. We thus have every reason to believe that the difference we find here would hold for unfamiliar pictures
}

the N400 response to within category violations for words, and left hemisphere processing shows a decreased N400 response to these items relative to right hemisphere processing. In contrast, increased constraint increases the N400 response to between category violations for pictures, and left hemisphere processing correspondingly shows an increased N400 response to these items relative to right hemisphere processing. There thus seems to be a relationship between hemispheric differences and effects of contextual constraint (as has been observed in previous behavioral work, e.g. Faust and Kravetz [20]). We suggest that this relationship arises because sentential constraint primarily influences how effectively information in a context affords predictions about features of the item likely to appear next-and it is only the left hemisphere that uses context to preactivate.

More generally, these modality-based differences argue against strong versions of the common semantic systems hypothesis, suggesting instead that access to semantic memory is not modality-independent. We have suggested previously [23] that this difference may arise in part because of the increased specificity of the semantic information available from a picture. The specific semantic feature information readily available from a picture can help differentiate the contextually expected completion from globally similar items in the same semantic category, making facilitation of the within category violations less likely. Concomitantly, the increased specificity may create a greater barrier to the integration of inappropriate and less-related targets, and hence a larger N400 response to the between category violations (for a more extensive discussion of this issue, see [23]).

In addition to finding hemispheric differences in the $\mathrm{N} 400$ time window, we find effects of hemifield of presentation and ending type - and interactions between the two- on earlier ERP components, including the P2 and the N300. ERP differences as a function of ending type begin much earlier-in the time window of the P2 $(150-250 \mathrm{~ms})$-for right visual field (left hemisphere) presentations than for left visual field (right hemisphere) ones. P2 amplitude modulations have been linked to the detection/analysis of basic visual features (e.g. orientation, size, color) in selective attention tasks (e.g. $[30,43])$, with increased amplitudes observed in response to stimuli containing the attended target features. In this study, with presentation to the right visual field, we found larger P2 amplitudes in response to expected items and smaller P2 amplitudes to between category violations. In contrast, with presentation to the left visual field, P2 amplitudes did not vary with ending type. Only in the left hemisphere, then, does processing of context seem to provide top-down information allowing for more efficient visual feature extraction from targets (expected items) than from unexpected items.

as well-that it is a true effect of modality and not specific to repeated (familiar) pictures. There may be hemispheric differences in sensitivity to repetition, and one could imagine that this might interact with contextual congruency, but there would seem to be no basis for predicting that it would affect one violation type differently from the other. 
This is consistent with the idea that the left hemispherebut not the right-is using context predictively and suggests, further, that the left hemisphere uses context to make predictions about perceptual as well semantic features of upcoming items. $^{8}$

There are interesting correspondences between this pattern and previously reported hemispheric differences in visual processing indicating that the left hemisphere makes more use of structured, top-down information than does the right. For example, when processing spatial relations between objects, it has been found that the left hemisphere more efficiently evaluates "categorical" relations (e.g. above/below) while the right hemisphere more efficiently evaluates "metric" relations (e.g. absolute distance) (e.g. $[29,35,36])$. One crucial difference between these types of spatial relations is that the former is reference frame dependent (e.g. "above" becomes "below" if the view is rotated $180^{\circ}$ ) while the latter is not-and, in fact, it has been found that factors that affect one's ability to create a reference frame also affect the pattern of hemispheric differences observed in these type of tasks [1]. Thus, the left hemisphere's processing of spatial information seems to be more dependent on having top-down structure-here, information about the appropriate reference frame-than does the right. The use of a reference frame seems to characterize aspects of each hemisphere's object recognition capabilities as well. Right hemisphere damage is associated with difficulties recognizing objects from unconventional viewpoints (e.g. $[67,68])$. Interestingly, it has been suggested that such difficulties may stem from the inappropriate use of reference frame information by the intact left hemisphere of these patients. Humphreys and Riddoch [33] have suggested, for example, that when viewing photographs of objects from unusual views, patients with right hemisphere damage impose the two-dimensional reference frame created by the border of the photograph ${ }^{9}$ onto the object contained therein and thus fail to recognize that a rotation in depth has occurred. They showed that when depth cues were provided or when the objects were skewed with respect to the photograph border (a cue that the reference frame of the border was inappropriate), these patients' performance improved considerably. Thus, left hemisphere object recognition, like left hemisphere spatial processing, seems to be guided by top-down information in the form of predictions-"guesses"-about the appropriate frame of reference. Clearly more work remains to be done, but there are intriguing indications that the left hemisphere's tendency to use a predictive, knowledge-driven processing strategy may extend beyond the domain of language comprehension.

\footnotetext{
${ }^{8}$ The P2 window was not examined separately in the word experiment, in part because there it was difficult to determine where $\mathrm{P} 2$ effects ended and N400 effects began. However, to the extent that there are P2 effects in the word data, they go in the same direction as those observed herewith differences for right but not left visual field presentations.

${ }^{9}$ This is the canonical reference frame for viewing photographs and, in most cases, an appropriate one.
}

With left hemisphere initiated processing, the ending type differences that begin in the $\mathrm{P} 2$ time window then continue in the N300 time window $(250-350 \mathrm{~ms})$. In contrast, with right hemisphere initiated processing, ending type differences are first observed in the N300 time window. In this time window we observe neither a main effect of hemifield nor an interaction of hemifield and violation type. For presentation in both hemifields, responses to within category violations were more positive than responses to between category violations. Responses to within category violations did not differ as a function of hemifield, while responses to between category violations showed a non-significant trend to be larger with presentation to the right visual field (left hemisphere) than with presentation to the left visual field (right hemisphere). By $300 \mathrm{~ms}$, therefore, processing in both hemispheres is affected by a picture's fit to context and, simultaneously, the categorical relationship between the picture and the item most expected in the context. As already discussed, these similar effects (as a function of hemifield) in the N300 time window then give way to a different pattern of effects in the N400 time window. Whereas all three ending types continue to be differentiated with right visual field (left hemisphere) presentations, the difference between the ending types is reduced or eliminated with left visual field (right hemisphere) presentations, leaving only a congruency effect in the later (N400) time window.

Such differences in the pattern of response as a function of ending type and hemifield in the two time windows support the argument that there are, indeed, two separable components elicited by pictures: an early, more frontally-distributed negativity that Holcomb and McPherson [32] called the N300 and a later negativity with a more central-posterior distribution that is analogous to the N400 component typically observed for words. Using a picture-picture priming task, McPherson and Holcomb [46] found evidence that the N300 and $\mathrm{N} 400$ were differentially sensitive to semantic variables: while the N400 response was graded by the strength of the relationship between the pictures in the pair, the N300 response differentiated only unrelated from related pictures and did not differentiate highly from moderately-related pairs (and, as already mentioned, in other work they found that the N300 but not the N400 was sensitive to object structure [45]). In our previous study of picture processing in sentence contexts (with central presentation), we did not find differences in the pattern of response to the three ending types in the N300 as compared with the N400 time windows [23]. Here, however, we find that the two components are differentially sensitive to hemifield of presentation, providing further support for the idea that they index somewhat different processes mediated by at least partially non-overlapping sets of neural generators.

McPherson and Holcomb [46] have speculated that the N300 may index processes specifically associated with the high-level analysis of imagistic representations. If so, these results may be taken to suggest that object recognition can be facilitated by top-down information from a congruent 
context and also by categorical similarity-perhaps resulting from the fact that items in the same category often share key visual features. Visual similarity also may help explain why we observed an effect of categorical relatedness on the N300 response, while McPherson and Holcomb [46] found that the N300 was not sensitive to degree of semantic relatedness. Whereas we used categorically-related stimuli, which will often tend to share visual features, McPherson and Holcomb's study included associatively-related items (e.g. hamburger and fries), which may be less similar at a visual level. ${ }^{10}$

In this study and in our previous word study we showed that the right hemisphere's use of sentence context information does not make it more prepared to process the conceptual features of the within category violations than of the between category violations, while the left hemisphere's strategy does. In this study, we also showed that only the left hemisphere's strategy facilitates the extraction of basic visual feature information (indexed as changes in P2 amplitude) from the more expected pictures. The N300 data, however, show that the right hemisphere's use of context does seem to make it more prepared to process the within category violations at some (higher-order visual processing?) level. We suspect that this is specifically because of the visual similarity—and hence confusability—of our expected exemplar and within category violation pictures. In future research, it will prove interesting to compare the processing of visually but not conceptually-related items with that to conceptually but not visually-related items and thereby to gain a more complete picture of how the two hemispheres extract information from sentence contexts and from pictures on-line.

In conclusion, then, there seems to be a difference in how the two cerebral hemispheres use context to facilitate the semantic processing of an item, and this difference seems to be somewhat independent of modality. As was true for word processing [21], we found that the semantic analysis of a picture when initiated by the right hemisphere was driven primarily by the plausibility of that item in the specific sentence context. In contrast, the semantic processing of pictures when initiated by the left hemisphere was affected by both fit to context and the semantic similarity of the item to that most predicted in the context. The context-independent organization of information in semantic memory thus seems to have a larger impact on both picture and word processing in the left than in the right hemisphere. This seems to be a general difference across the hemispheres that holds regardless of whether the stimulus being integrated into a sentence context is a word or a picture. We thus do not find evidence here to suggest that the hemispheres differ in the extent to

\footnotetext{
${ }^{10}$ There were many other differences between the two studies as well which could impact the nature of the N300 response: central versus peripheral presentation, pictures versus line drawings, picture pairs versus sentence contexts, relatedness-judgement task versus reading for comprehension, etc. More research will obviously be needed to determine exactly what variables the $\mathrm{N} 300$ is sensitive to and under what conditions.
}

which they can successfully extract semantic information from words versus pictures (of the type used here) (see, e.g. [9]); rather, there seems to be a more basic difference in how the hemispheres use context information to prepare for the processing of any upcoming meaningful stimulus.

While the N400 congruity effects observed for picture processing as a function of visual field of presentation were overall similar to those previously observed for words, we also found modality-based processing differences. Picture processing in both hemispheres was associated with an earlier component, the $\mathrm{N} 300$, that-unlike the pattern observed for the N400-was unaffected by visual field of input. Furthermore, while the overall pattern of N400 effects in each hemisphere was similar for pictures as for words, in the case of pictures it was the response to between category violations that varied in amplitude with visual field of presentation, while in the case of words it was the response to within category violations that differed. Thus, semantic processing does not seem to be completely modality-independent. Instead, the results of this study and those that preceded it suggest that the semantic information derived from a sensory stimulus is a function of both the nature of the stimulus (e.g. whether it is a word or a picture) and the hemisphere that initiates its processing.

\section{Acknowledgements}

The research reported here was supported by a Howard Hughes Predoctoral Fellowship to K.F. and Grants HD22614, AG08313, and MH52893 to M.K.

\section{References}

[1] Banich MT, Federmeier KD. Categorical and metric spatial processes distinguished by task demands and practice. Journal of Cognitive Neuroscience 1999;11:153-66.

[2] Barrett SE, Rugg MD. Event-related potentials and the semantic matching of pictures. Brain Cognition 1990;14:201-12.

[3] Beeman M. Coarse semantic coding and discourse comprehension. In: Beeman $\mathrm{M}$, Chiarello $\mathrm{C}$, editors. Right hemisphere language comprehension: perspectives from cognitive neuroscience. Mahwah, NJ: Lawrence Erlbaum Associates, 1998. p. 255-84.

[4] Biederman I, Cooper EE. Object recognition and laterality: null effects. Neuropsychologia 1991;29:685-94.

[5] Burgess C, Lund K. Modeling cerebral asymmetries in high-dimensional space. In: Beeman M, Chiarello C, editors. Right hemisphere language comprehension: perspectives from cognitive neuroscience. Mahwah, NJ: Lawrence Erlbaum Associates, 1998. p. 215-44.

[6] Burgess C, Simpson GB. Cerebral hemispheric mechanisms in the retrieval of ambiguous word meanings. Brain Language 1988;33:86103.

[7] Caramazza A, Hillis AE, Rapp BC, Romani C. The multiple semantics hypothesis: multiple confusions? Cognitive Neuropsychology 1990;7:161-89.

[8] Chiarello C. Interpretation of word meanings by the cerebral hemispheres: one is not enough. In: Schwanenflugel PJ, editor. The psychology of word meanings. Hillsdale, NJ: Lawrence Erlbaum Associates, 1991. p. 251-78. 
[9] Chiarello C. On codes of meaning and the meaning of codes: semantic access and retrieval within and between hemispheres. In: Beeman $\mathrm{M}$, Chiarello C, editors. Right hemisphere language comprehension: perspectives from cognitive neuroscience. Mahwah, NJ: Lawrence Erlbaum Associates, 1998. p. 141-60.

[10] Chiarello C, Burgess C, Richards L, Pollock A. Semantic and associative priming in the cerebral hemispheres: some words do, some words do not ... sometimes, some places. Brain Language 1990;38:75-104.

[11] Chiarello C, Liu S, Faust M. Bihemispheric sensitivity to semantic anomaly. Neuropsychologia 2001;39:1451-63.

[12] Chiarello C, Richards L. Another look at categorical priming in the cerebral hemispheres. Neuropsychologia 1992;30:381-92.

[13] Chiarello C, Senehi J, Soulier M. Viewing conditions and hemisphere asymmetry for the lexical decision. Neuropsychologia 1986;24: 521-9.

[14] Christman S. Perceptual characteristics in visual laterality research. Brain Cognition 1989;11:238-57.

[15] Christman SD. On the complex relation between perceptual characteristics and hemispheric asymmetry. Brain Cognition 1993;21:123-9.

[16] Coney J, Abernethy M. Picture-name priming in the cerebral hemispheres. Brain Language 1994;47:300-13.

[17] Cronin-Golomb A. Comprehension of abstract concepts in right and left hemispheres of complete commissurotomy subjects. Neuropsychologia 1986;24:881-7.

[18] Delis DC, Robertson LC, Efron R. Hemispheric specialization of memory for visual hierarchical stimuli. Neuropsychologia 1986;24:205-14.

[19] Faust M, Babkoff H, Kravetz S. Linguistic processes in the two cerebral hemispheres: implications for modularity versus interactionism. Journal of Clinical Experimental Neuropsychology 1995; 17:171-92.

[20] Faust M, Kravetz S. Levels of sentence constraint and lexical decision in the two hemispheres. Brain Language 1998;62:149-62.

[21] Federmeier KD, Kutas M. Right words and left words: electrophysiological evidence for hemispheric differences in meaning processing. Cognitive Brain Research 1999;8:373-92.

[22] Federmeier KD, Kutas M. A rose by any other name: long-term memory structure and sentence processing. Journal of Memory Language 1999;41:469-95.

[23] Federmeier KD, Kutas M. Meaning and modality: influences of context, semantic memory organization, and perceptual predictability on picture processing. Journal of Experimental Psychology: Learning, Memory, Cognition 2001;27:202-24.

[24] Fink GR, Halligan PW, Marshall JC, Frith CD, Frackowiak RSJ, Dolan RJ. Where in the brain does visual attention select the forest and the trees? Nature 1996;382:626-8.

[25] Galaburda AM. Anatomic basis of cerebral dominance. In: Davidson RJ, Hugdahl K, editors. Brain asymmetry. Cambridge: MIT Press, 1995. p. 51-73.

[26] Galaburda AM, Aboitiz F, Rosen GD, Sherman GF. Histological asymmetry in the primary visual cortex of the rat: implications for mechanisms of cerebral asymmetry. Cortex 1986;22:151-60.

[27] Ganis G, Kutas M, Sereno MI. The search for 'common sense': an electrophysiological study of the comprehension of words and pictures in reading. Journal of Cognitive Neuroscience 1996;8:89-106.

[28] Glaser WR. Picture naming: lexical access in speech production [special issue]. Cognition 1992;42:61-105.

[29] Hellige JB, Michimata C. Categorization versus distance: hemispheric differences for processing spatial information. Memory Cognition 1989;17:770-6.

[30] Hillyard SA, Muente TF. Selective attention to color and location: an analysis with event-related brain potentials. Perception Psychophysics 1984;36:185-98.

[31] Hines D, Sawyer PK, Dura J, Gilchrist J, Czerwinski M. Hemispheric asymmetry in use of semantic category information. Neuropsychologia 1984;22:427-33.
[32] Holcomb PJ, McPherson WB. Event-related brain potentials reflect semantic priming in an object decision task. Brain Cognition 1994;24:259-76.

[33] Humphreys GW, Riddoch MJ. Routes to object constancy: implications from neurological impairments of object constancy. Quarterly Journal of Experimental Psychology: Human Experimental Psychology 1984;36A:385-415.

[34] Koivisto M. Time course of semantic activation in the cerebral hemispheres. Neuropsychologia 1997;35:497-504.

[35] Kosslyn SM, Chabris CF, Marsolek CJ, Koenig O. Categorical versus coordinate spatial relations: computational analyses and computer simulations. Journal of Experimental Psychology: Human Perception Performance 1992;18:562-77.

[36] Kosslyn SM, Koenig O, Barrett A, Cave CB, Tang J, Gabrieli JDE. Evidence for two types of spatial representations: hemispheric specialization for categorical and coordinate relations. Journal of Experimental Psychology: Human Perception Performance 1989;15:723-35.

[37] Kutas M, Federmeier KD. Electrophysiology reveals semantic memory use in language comprehension. Trends Cognitive Science 2001;4:463-70.

[38] Kutas M, Van Petten CK. Electrophysiological perspectives on comprehending written language. In: Rossini PM, Maugiere F, editors. New trends and advanced techniques in clinical neurophysiology. Amsterdam: Elsevier, 1990. p. 155-67.

[39] Kutas M, Van Petten CK. Psycholinguistics electrified: event-related brain potential investigations. In: Gernsbacher MA, editor. Handbook of psycholinguistics. San Diego: Academic Press, 1994. p. 83-143.

[40] Laeng B. Lateralization of categorical and coordinate spatial functions: a study of unilateral stroke patients. Journal of Cognitive Neuroscience 1994;6:189-203

[41] Levine SC, Banich MT. Lateral asymmetries in the naming of words and corresponding line drawings. Brain Language 1982;17:34-43.

[42] Lewis DW, Diamond MC. The influence of gonadal steroids on the asymmetry of the cerebral cortex. In: Davidson RJ, Hugdahl K, editors. Brain asymmetry. Cambridge, MA: MIT Press, 1995. p. 31-50.

[43] Luck SJ, Hillyard SA. Electrophysiological correlates of feature analysis during visual search. Psychophysiology 1994;31:291-308.

[44] McCarthy G, Wood CC. Scalp distributions of event-related potentials: an ambiguity associated with analysis of variance models. Electroencephalography Clinical Neurophysiology 1985;62:203-8.

[45] McPherson WB, Holcomb PJ. Semantic priming with pictures and the N400 component. Psychophysiology 1992;29:S51.

[46] McPherson WB, Holcomb PJ. An electrophysiological investigation of semantic priming with pictures of real objects. Psychophysiology 1999;36:53-65.

[47] Moscovitch M. Laterality and visual masking: interhemispheric communication and the locus of perceptual asymmetries for words. Canadian Journal of Psychology 1983;37:85-106.

[48] Neville HJ, Kutas M, Schmidt A. Event-related potential studies of cerebral specialization during reading. I. Studies of normal adults. Brain Language 1982;16:300-15.

[49] Nigam A, Hoffman JE, Simons RF. N400 to semantically anomalous pictures and words. Journal of Cognitive Neuroscience 1992;4:15-22.

[50] Oldfield RC. The assessment and analysis of handedness: the Edinburgh inventory. Neuropsychologia 1971;9:97-113.

[51] Paivio A. Imagery and verbal processes. New York: Holt, 1971

[52] Paivio A. Mental representations: a dual coding approach. New York: Oxford University Press, 1986.

[53] Paivio A. Dual coding theory: retrospect and current status. Canadian Journal of Psychology 1991;45:255-87.

[54] Potter MC, Faulconer BA. Time to understand pictures and words. Nature 1975;253:437-8.

[55] Potter MC, Kroll JF, Yachzel B, Carpenter E, Sherman J. Pictures in sentences: understanding without words. Journal of Experimental Psychology: General 1986;115:281-94. 
[56] Pylyshyn ZW. Computation and cognition: issues in the foundations of cognitive science. Behavioral Brain Science 1980;3:111-32.

[57] Reuter-Lorenz PA, Baynes K. Modes of lexical access in the callosotomized brain. Journal Cognitive Neuroscience 1992;4: 155-64.

[58] Riddoch MJ, Humphreys GW, Coltheart M, Funnell E. Semantic systems or system? Neuropsychological evidence re-examined. The cognitive neuropsychology of visual and semantic processing of concepts [special issue]. Cognitive Neuropsychology 1988;5:3-25.

[59] Rosch E, Mervis CB, Gray WD, Johnson DM, Boyes-Braem P. Basic objects in natural categories. Cognitive Psychology 1976;8:382-439.

[60] Rybash JM, Hoyer WJ. Hemispheric specialization for categorical and coordinate spatial representations: a reappraisal. Memory Cognition 1992;20:271-6.

[61] Scheibel AB, Paul LA, Fried I, Forsythe AB, Tomiyasu U, Wechsler $A$, et al. Dendritic organization of the anterior speech area. Experimental Neurology 1985;87:109-17.

[62] Shallice T. Specialization within the semantic system. The cognitive neuropsychology of visual and semantic processing of concepts [special issue]. Cognitive Neuropsychology 1988;5:133-42.

[63] Snodgrass JG, McCullough B. The role of visual similarity in picture categorization. Journal of Experimental Psychology: Learning, Memory, Cognition 1986;12:147-54.
[64] Snodgrass JG, Vanderwart M. A standardized set of 260 pictures: norms for name agreement, image agreement, familiarity, and visual complexity. Journal of Experimental Psychology: Human Learning Memory 1980;6:174-215.

[65] Tucker DM, Williamson PA. Asymmetric neural control systems in human self-regulation. Psychology Review 1984;91:185-215.

[66] Van Kleeck MH. Hemispheric differences in global versus local processing of hierarchical visual stimuli by normal subjects: new data and a meta-analysis of previous studies. Neuropsychologia 1989;27:1165-78

[67] Warrington EK, James M. Visual object recognition in patients with right hemisphere lesions: axes or features? Perception 1986;15: $355-66$.

[68] Warrington EK, Taylor AM. Two categorical stages of object recognition. Perception 1978;7:695-705.

[69] Zaidel DW. Hemispheric asymmetry in long-term semantic relationships. Cognitive Neuropsychology 1987;4:321-32.

[70] Zaidel DW. Hemispheric asymmetry in memory for pictorial semantics in normal subjects. Neuropsychologia 1987;25:487-95.

[71] Zaidel DW. Hemifield asymmetries in memory for incongruous scenes. Cortex 1988;24:231-44.

[72] Zaidel E. Performance on the Itpa following cerebral commissurotomy and hemispherectomy. Neuropsychologia 1979;17:259-80. 